\title{
Intuitionistic Fuzzy Transportation Problem by Zero Point Method
}

\author{
Velichka Traneva \\ "Prof. Asen Zlatarov" University \\ "Prof. Yakimov" Blvd, Burgas 8000, Bulgaria \\ Email: veleka13@gmail.com
}

\author{
Stoyan Tranev \\ "Prof. Asen Zlatarov" University \\ "Prof. Yakimov" Blvd, Burgas 8000, Bulgaria \\ Email: tranev@abv.bg
}

\begin{abstract}
The transportation problems (TPs) support the optimal management of the transport deliveries. In classical TPs the decision maker has information about the crisp values of the transportation costs, availability and demand of the products. Sometimes in the parameters of TPs in real life there is ambiguity and vagueness caused by uncontrollable market factors.

Uncertain values can be represented by fuzzy sets (FSs) of Zadeh. The FSs have the degrees of membership and nonmembership. The concept of intuitionistic fuzzy sets (IFSs) originated in 1983 as an extension of FSs. Atanasov's IFSs also have a degree of hesitansy to representing the obscure environment.

In this paper we formulate the $\mathbf{T P}$, in which the transportation costs, supply and demand values are intuitionistic fuzzy pairs (IFPs), depending on the diesel prices, road condition, weather and other factors. Additional constraints are included in the problem: limits for the transportation costs. Its main objective is to determine the quantities of delivery from producers to buyers to maintain the supply and demand requirements at the cheapest transportation costs. The aim of the paper is to extend the fuzzy zero point method (FZPM [35]) to the intuitionistic FZPM (IFZPM) to find an optimal solution of the intuitionistic fuzzy TP (IFTP) using the IFSs and index matrix (IM) concepts, proposed by Atanassov. The solution algorithm is demonstrated by a numerical example. Its optimal solution is compared with that obtained by the intuitionistic fuzzy zero suffix method (IFZSM).
\end{abstract}

\section{INTRODUCTION}

$\mathbf{T}$ HE TP originally proposed by Hitchcock in 1941 [12]. Dantzig, in 1951, used simplex method to the TP [13] The first overall, finished method for solving TP ("method of potentials") is developed by Kantorovich in 1949 [26].

In classical TP the decision maker has information about the values of the transportation costs, the demanded and offered quantities of the product. In real-life transportation problems, some of its parameters are uncertain due to climatic, road conditions or other market conditions. The costs are fuzzy in the absence of information or in uncertain environment. Zadeh proposed the fuzzy set (FS) theory [27] in 1963 to deal with uncertainty. In 1983, Atanassov proposed the IFSs [17], which is an extension of FSs of Zadeh. The main difference between FSs and IFSs is that the IFSs have a degree of hesitancy.

This work on Sect. I and Sect. II is supported by the project of Asen Zlatarov University under Ref. No. NIX-423/2019 "Innovative methods for extracting knowledge management" The work on Sect. III and Sect. IV is supported by the Ministry of Education and Science under the Programme "Young scientists and postdoctoral students", approved by DCM \# 577/17.08.2018.
The following is a brief theoretical overview in the field of fuzzy (FTPs) or intuitionistic FTPs (IFTPs). Chanas et al., in 1984, has proposed a fuzzy linear programming model for solving TPs with clear transportation costs, fuzzy supply and demand values [39]. Gen et al. have given a genetic algorithm for finding an optimal solution of a bicriteria solid TP with fuzzy numbers (FNs) [28]. Jimenez and Verdegay, in 1999, researched fuzzy Solid TP with trapezoidal FNs and presented a genetic approach for solving FTP [11]. Liu and Kao [41] demostrated a method, based on Zadeh's extension principle, to find the optimal solution of the trapezoidal FTPs. Dinagar and Palanivel [9] have described fuzzy Vogel's approximation method and modified distribution method for determining an initial solution of trapezoidal FTPs. Pandian and Natarajan, in 2010, studied zero point method for solution for FTP with trapezoidal fuzzy parameters [35]. Improved zero point methods were described in (see [1], [2], [43]) for solving trapezoidal and triangular FTP.

Kaur and Kumar, in 2012, introduced fuzzy least cost method, fuzzy north west corner rule and fuzzy Vogel approximation method for determining of an optimal solution of FTP [5]. Basirzadeh [16] has found a fuzzy optimal solution of fully FTPs by transforming the fuzzy parameters into the crisp parameters using classical algorithms. Gani et al. [3] used Arsham and Khan's simplex algorithm [15] to find a fuzzy optimal solution of FTPs with trapezoidal fuzzy parameters. A comparative analysis on the FTPs [42] was made and the conclusion has given that the zero point method is better than both the modified distribution method and Vogel's Approximation method. Patil and Chandgude, in 2012, performed "Fuzzy Hungarian approach" for TP with trapezoidal FNs [7]. Aggarwal and Gupta, in 2013, described an procedure for solving intuitionistic fuzzy TP (IFTP) with trapezoidal IFNs via ranking method [14]. Jahihussain and Jayaraman, in 2013, presented a zero suffix method for obtaining an optimal solution for FTPs with triangular and trapezoidal FNs (see [37], [38]). Zero suffix method to solve FTP after its converting into the crisp problem was applied in [32] and [44]. A fuzzified version of zero suffix method was performed and applied in [29], in 2018, to FTPs. Shanmugasundari and Ganesan, in 2013, proposed a fuzzy modified distribution algorithm and a fuzzy approximation method of Vogel to solve FTP with FNs [30]. Gani and Abbas, in 2014 [4], and Kathirvel, and 
Balamurugun, in 2012 (see [24], [25]), proposed a method for solving TP in which the quantities demanded and offered are represented in the form of the trapezoidal intuitionistic FNs (IFNs). Antony et al. used Vogel's approximation method for solving triangular IFTP in 2014 [36]. "PSK method" for finding an optimal solution to IFTPs was presented by Kumar and Hussain in 2015 [33]. Fully FTPs was resolved in [40], in 2017, using a new method, based on the Hungarian and MODI algorithm. Two new methods for finding a fuzzy optimal solution of TPs with the LR flat fuzzy numbers were proposed by Kaur, Kacprzyk and Kumar [6], based on the tabular representation and on the fuzzy linear programming formulation. In [49], we have proposed for the first time the IFZSM to determine an optimal solution of the IFTP, interpreted by the IFSs and IMs [18] concepts.

Here, we proposed for the first time intuitionistic fuzzy zero point method (IFZPM) to solve optimally a type of TP, in which the transportation costs, supply and demand quantities are IFPs, depending on the climatic, road conditions and economic factors. The constraints are formulated to the problem additionally: limits to the transportation costs. The optimal solution algorithm is demonstrated with a numerical example. The optimal solutions, respectively obtained after the application of the intuitionistic fuzzy zero suffix method (IFZSM) and IFZPM, are compared. The two methods for finding an optimal solution for IFTPs are free from the problem of degeneracy. The optimal transportation cost of the studied TP, obtained by the IFZPM is better than or equal to that after the application of the IFZSM. The advantages of the algorithm are that it can be easy generalized for an application to multidimensional data and can be applied to both the TP with clear or known parameters, and with intuitionistic fuzzy ones. The structure of this paper is as follows: Section 2 recalls some remarks of the theories of the IMs and the IFPs. In Section 3, we propose an algorithm for IFTP extending the fuzzy zero point method [35] and using the concepts of IMs and IFSs. The reliability of the proposed approach is demonstrated by an example in Section 4 and the results are compared with those obtained after application of IFZSM. Section 5 outlines the conclusion and some directions for future research.

\section{INTRODUCTION TO IMS AND INTUITIONISTIC FUZZY LOGIC}

In this section we recall some basic definitions on intuitionistic fuzzy pairs from (see [10], [19], [21], [23], [46]) and on index matrix apparatus from (see [20], [48]).

\subsection{Short Remarks on Intuitionistic Fuzzy (IF) Logic}

The IFP has the form of an ordered pair $\langle a, b\rangle=$ $\langle\mu(p), v(p)\rangle$, where $a, b \in[0,1]$ and $a+b \leq 1$, that is used as an evaluation of a proposition $p$ (see [21], [23]). $\mu(p)$ and $v(p)$ respectively determine the "truth degree" (degree of membership) and "falsity degree" (degree of nonmembership).
Let us recall some basic operations as "negation", "addition", "subtraction", "multiplication" over two IFPs $x=\langle a, b\rangle$ and $y=\langle c, d\rangle$.

$$
\begin{gathered}
\neg x=\langle b, a\rangle ; \\
x \wedge_{1} y=\langle\min (a, c), \max (b, d)\rangle ; \\
x \vee_{1} y=\langle\max (a, c), \min (b, d)\rangle ; \\
x \wedge_{2} y=x+y=\langle a+c-a \cdot c, b . d\rangle ; \\
x \vee_{2} y=x \cdot y=\langle a \cdot c, b+d-b \cdot d\rangle ; \\
\alpha \cdot x=\left\langle 1-(1-a)^{\alpha}, b^{\alpha}\right\rangle(\alpha \in R) ; \\
x-y=\langle\max (0, a-c), \min (1, b+d, 1-a+c)\rangle .
\end{gathered}
$$

The forms of the relations with IFPs are the following

$$
\begin{array}{cc}
x \geq y \text { iff } a \geq c \text { and } b \leq d ; & x \leq y \text { iff } a \leq c \text { and } b \geq d ; \\
x \geq \square y \text { iff } a \geq c ; & x \leq_{\square} y \text { iff } a \leq c ; \\
x \geq_{\diamond} y \text { iff } b \leq d ; & x \leq_{\diamond} y \text { iff } b \geq d ; \\
x=y & \text { iff } a=c \text { and } b=d \\
x \geq_{R} y & \text { iff } R_{\langle a, b\rangle} \leq R_{\langle c, d\rangle},
\end{array}
$$

where

$$
R_{\langle a, b\rangle}=0.5(2-a-b) 0.5(|1-a|+|b|+|1-a-b|)[10] .
$$

The IFP $x$ is an "intuitionistic fuzzy false pair" (IFFP) if and only if $a \leq b$, while $x$ is a "false pair" (FP) iff $a=$ $0, b=1$.

Let a set $E$ be fixed. An "intuitionistic fuzzy set" (IFS) $A$ in $E$ is an object of the following form (see [19]):

$$
A=\left\{\left\langle x, \mu_{A}(x), v_{A}(x)\right\rangle \mid x \in E\right\},
$$

where $\mu_{A}: E \rightarrow[0,1]$ and $v_{A}: E \rightarrow[0,1]$ define the degrees of membership and non-membership of the $x \in E$, respectively, and $0 \leq \mu_{A}(x)+v_{A}(x) \leq 1$ for every $x \in E$ :

2.2. Definition, Operations and Relations over Intuitionistic Fuzzy Index Matrices

Let $\mathscr{I}$ be a fixed set. The definition of two-dimensional intuitionistic fuzzy index matrix (2-D IFIM) with index sets $K$ and $L(K, L \subset \mathscr{I})$ is the following:

\begin{tabular}{c|ccccc}
\multicolumn{6}{c}{$\left[K, L,\left\{\left\langle\mu_{k_{i}, l_{j}}, v_{k_{i}, l_{j}}\right\rangle\right\}\right]$} \\
\\
\hline$k_{1}$ & $l_{1}$ & $\ldots$ & $l_{j}$ & $\ldots$ & $l_{n}$ \\
\hline$\vdots$ & $\vdots$ & $\ddots$ & $\vdots$ & $\ddots$ & $\vdots$ \\
$k_{m}$ & $\left\langle\mu_{\left.k_{1}, l_{1}, l_{1}, v_{k_{1}, l_{1}}\right\rangle}, v_{k_{m}, l_{1}}\right\rangle$ & $\ldots$ & $\left\langle\mu_{k_{1}, l_{j}}, v_{k_{1}, l_{j}}\right\rangle$ & $\ldots$ & $\left\langle\mu_{k_{1}, l_{n}}, v_{k_{1}, l_{n}}\right\rangle$ \\
\hline$\left.k_{k_{m}, l_{j}}, v_{k_{m}, l_{j}}\right\rangle$ & $\ldots$ & $\left\langle\mu_{k_{m}, l_{n}}, v_{k_{m}, l_{n}}\right\rangle$
\end{tabular}

where for $i=1, \ldots, m ; j=1, \ldots, n$ :

$$
0 \leq \mu_{k_{i}, l_{j}}, v_{k_{i}, l_{j}}, \mu_{k_{i}, l_{j}}+v_{k_{i}, l_{j}} \leq 1 .
$$

The basic operations over two IMs

$$
A=\left[K, L,\left\{\left\langle\mu_{k_{i}, l_{j}}, v_{k_{i}, l_{j}}\right\rangle\right\}\right]
$$

and

$$
B=\left[P, Q,\left\{\left\langle\rho_{p_{r}, q_{s}}, \sigma_{p_{r}, q_{s}}\right\rangle\right\}\right]
$$

are as follows [20]: 
Negation: $\neg A=\left[K, L,\left\{\left\langle v_{k_{i}, l_{j}}, \mu_{k_{i}, l_{j}}\right\rangle\right\}\right]$.

Addition-(o,*): $A \oplus_{(\circ, *)} B=\left[K \cup P, L \cup Q,\left\{\left\langle\phi_{t_{u}, v_{w}}, \psi_{t_{u}, v_{w}}\right\rangle\right\}\right]$, where $\left\langle\phi_{t_{u}, v_{w}}, \psi_{t_{u}, v_{w}}\right\rangle$

$$
= \begin{cases}\left\langle\mu_{k_{i}, l_{j}}, v_{k_{i}, l_{j}}\right\rangle, & \text { if } t_{u}=k_{i} \in K \text { and } v_{w}=l_{j} \in L-Q \\ \left\langle\rho_{p_{r}, q_{s}}, \sigma_{p_{r}, q_{s}}\right\rangle, & \text { or } t_{u}=k_{i} \in K-P \text { and } v_{w}=l_{j} \in L ; \\ & \text { or } t_{u}=p_{r} \in P \text { and } v_{w}=q_{s} \in Q-L \\ & \text { and } v_{w}=q_{s} \in Q ; \\ \left\langle\circ\left(\mu_{k_{i}, l_{j}}, \rho_{p_{r}, q_{s}}\right),\right. & \text { if } t_{u}=k_{i}=p_{r} \in K \cap P \\ \left.*\left(v_{k_{i}, l_{j}}, \sigma_{p_{r}, q_{s}}\right)\right\rangle, & \text { and } v_{w}=l_{j}=q_{s} \in L \cap Q ; \\ \langle 0,1\rangle, & \text { otherwise. }\end{cases}
$$

where $\langle\circ, *\rangle \in\{\langle\max , \min \rangle,\langle\min , \max \rangle,\langle$ average, average $\rangle\}$.

Termwise subtraction-(max,min):

$$
A-{ }_{(\max , \min )} B=A \oplus_{(\max , \min )} \neg B .
$$

Termwise multiplication- $(\min , \max )$ :

$$
A \otimes_{(\min , \max )} B=\left[K \cap P, L \cap Q,\left\{\left\langle\phi_{t_{u}, v_{w}}, \psi_{t_{u}, v_{w}}\right\rangle\right\}\right],
$$

where

$$
\left\langle\phi_{t_{u}, v_{w}}, \psi_{t_{u}, v_{w}}\right\rangle=\left\langle\min \left(\mu_{k_{i}, l_{j}}, \rho_{p_{r}, q_{s}}\right), \max \left(v_{k_{i}, l_{j}}, \sigma_{p_{r}, q_{s}}\right)\right\rangle .
$$

Transposition: $A^{\prime}$ is the transposed IM of $A$.

Reduction: The symbol " $\perp$ " denotes the lack of some component in the definitions. The operation $(k, \perp)$-reduction of the IM $A$ is defined by:

$$
A_{(k, \perp)}=\left[K-\{k\}, L,\left\{c_{t_{u}, v_{w}}\right\}\right],
$$

where $c_{t_{u}, v_{w}}=a_{k_{i}, l_{j}}$ for $t_{u}=k_{i} \in K-\{k\}$ and $v_{w}=l_{j} \in L$. Projection: Let $M \subseteq K$ and $N \subseteq L$. Then,

$$
\operatorname{pr}_{M, N} A=\left[M, N,\left\{b_{k_{i}, l_{j}}\right\}\right],
$$

where for each $k_{i} \in M$ and each $l_{j} \in N, b_{k_{i}, l_{j}}=a_{k_{i}, l_{j}}$.

Substitution: Let IM $A=\left[K, L,\left\{a_{k, l}\right\}\right]$ be given. The some forms of the substitution over $A$ are defined for the couples of indices $(p, k)$ and/or $(q, l)$, respectively, by

$$
\begin{aligned}
& {\left[\frac{p}{k} ; \perp\right] A=\left[(K-\{k\}) \cup\{p\}, L,\left\{a_{k, l}\right\}\right],} \\
& {\left[\perp ; \frac{q}{l}\right] A=\left[K,(L-\{l\}) \cup\{q\},\left\{a_{k, l}\right\}\right] .}
\end{aligned}
$$

\section{Index type operations:}

$$
\begin{gathered}
\operatorname{AGIndex}_{\left\{(\min / \max ) /\left(\min _{\square} / \max _{\square}\right) /\left(\min _{\diamond} / \max _{\diamond}\right)\left(\min _{R} / \max _{R}\right)\right\}(\not)}(A) \\
=\left\langle k_{i}, l_{j}\right\rangle
\end{gathered}
$$

finds the index of the minimum/ maximum element of $A$ with no empty value in accordance with the relations (2).

AGIndex $\left\{(\min / \max ) /\left(\min _{\square} / \max _{\square}\right) /\left(\min _{\diamond} / \max _{\diamond}\right)\left(\min _{R} / \max _{R}\right)\right\}(\not \not)(\notin F)$

$$
(A)=\left\langle k_{i}, l_{j}\right\rangle
$$

presents the index of the minimum/ maximum element between the elements of $A$, whose indexes $\notin F$, with no empty value in accordance with the relations (2).

$$
\begin{gathered}
\left.\left.\operatorname{Index}_{\{(\min / \max ) /(\min \square} / \max _{\square}\right) /\left(\min _{\diamond} / \max _{\diamond}\right)\left(\min _{R} / \max _{R}\right)\right\}(\not), k_{i}(A) \\
=\left\{\left\langle k_{i}, l_{v_{1}}\right\rangle, \ldots,\left\langle k_{i}, l_{v_{x}}\right\rangle, \ldots,\left\langle k_{i}, l_{v_{V}}\right\rangle\right\},
\end{gathered}
$$

where $\left\langle k_{i}, l_{v_{x}}\right\rangle$ (for $\left.i=1, \ldots, m ; j=1, \ldots, n ; x=1, \ldots, V\right)$ are the indices of the minimum/ maximum IFFP of $k_{i}$-th row of $A$ with no empty value in accordance with the relations (2).

$$
\operatorname{Index}_{(\not)}(A)=\left\{\left\langle k_{1}, l_{v_{1}}\right\rangle, \ldots,\left\langle k_{i}, l_{v_{i}}\right\rangle, \ldots,\left\langle k_{m}, l_{v_{m}}\right\rangle\right\},
$$

where $\left\langle k_{i}, l_{v_{i}}\right\rangle$ (for $1 \leq i \leq m$ ) are the indices of the element of $A$, whose cell is full.

$$
\left.\operatorname{Index}_{(\max } \mu(v)\right), k_{i}(A)=\left\{\left\langle k_{i}, l_{v_{1}}\right\rangle, \ldots,\left\langle k_{i}, l_{v_{x}}\right\rangle, \ldots,\left\langle k_{i}, l_{v_{V}}\right\rangle\right\},
$$

where $\left\langle k_{i}, l_{v_{x}}\right\rangle$ (for $1 \leq i \leq V, 1 \leq x \leq n$ ) is the indices of the IFFP of $k_{i}$-th row of $A$, for which $\mu(v)_{k_{i}, l_{v_{x}}}$ is maximum.

$$
\left.\operatorname{Index}_{(\max } \mu(v)\right), l_{j}(A)=\left\{\left\langle k_{w_{1}}, l_{j}\right\rangle, \ldots,\left\langle k_{w_{y}}, l_{j}\right\rangle, \ldots,\left\langle k_{w_{W}}, l_{j}\right\rangle\right\},
$$

where $\left\langle k_{w_{y}}, l_{j}\right\rangle$ (for $1 \leq y \leq W, 1 \leq j \leq n$ ) are the indices of the IFFP of $l_{j}$-th column of $A$, for which $\mu(v)_{k_{w},} l_{j}$ is maximum. Aggregation operations

Let us use the operations $\#_{q},(q \leq i \leq 3)$ from [47] for scaling aggregation operations over two IFPs $x=\langle a, b\rangle$ and $y=\langle c, d\rangle$ : $x \#_{1} y=\langle\min (a, c), \max (b, d)\rangle$;

$x \#_{2} y=\langle$ average $(a, c)$, average $(b, d)\rangle$;

$x \#_{3} y=\langle\max (a, c), \min (b, d)\rangle$.

The following inequality holds:

$$
x \#_{1} y \leq x \#_{2} y \leq x \#_{3} y \quad[47] .
$$

Let $k_{0} \notin K$ be a fixed index. The definition of the aggregation operation by the dimension $K$ is [20], [47]: is:

$$
\begin{aligned}
& \alpha_{K, \#_{q}}\left(A, k_{0}\right)
\end{aligned}
$$

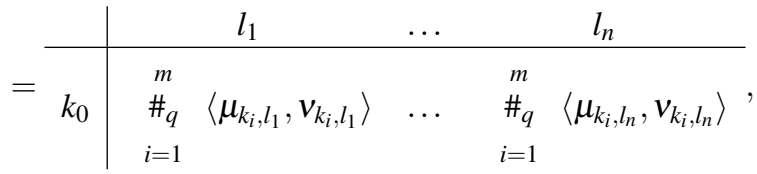

where $1 \leq q \leq 3$.

Aggregate global internal operation: $A G I O_{\oplus_{(\max , \min )}}(A)$. This operation finds the addition of all elements of $A$. Internal subtraction of the components of the IM $A$ ([45], [46], [48]):

$$
\begin{gathered}
I O_{-(\max , \min )}\left(\left\langle k_{i}, l_{j}, A\right\rangle,\left\langle p_{r}, q_{s}, B\right\rangle\right)=\left[K, L,\left\{\left\langle\gamma_{t_{u}, v_{w}}, \delta_{t_{u}, v_{w}}\right\rangle\right\}\right] \\
\qquad \begin{array}{ll}
\left\langle\gamma_{t_{u}, v_{w}}, \delta_{t_{u}, v_{w}}\right\rangle & \text { if } t_{u} \neq k_{i} \in K, \\
\left\langle\mu_{t_{u}, v_{w}}, v_{t_{u}, v_{w}}\right\rangle, & v_{w} \neq l_{j} \in L ; \\
\left\langle\max \left(0, \mu_{k_{i}, l_{j}}-\rho_{p_{r}, q_{s}}\right),\right. & \text { if } t_{u}=k_{i} \in K, \\
\left.\min \left(1, v_{k_{i}, l_{j}}+\sigma_{p_{r}, q_{s}}, 1-\mu_{k_{i}, l_{j}}+\rho_{p_{r}, q_{s}}\right)\right\rangle & v_{w}=l_{j} \in L
\end{array}
\end{gathered}
$$

where $k_{i} \in K, \quad l_{j} \in L ; \quad p_{r} \in P, \quad q_{s} \in Q$.

The non-strict relation "inclusion about value" The form of this type of relations between two IMs $A$ and $B$ is as follows: $A \subseteq_{v} B$ iff $(K=P) \&(L=Q) \&(\forall k \in K)(\forall l \in L)\left(a_{k, l} \leq b_{k, l}\right)$. 


\section{INTUITIONISTIC FUZZY ZERO POINT APPROACH TO THE IFTP}

Let us extend the IFTP from [49]: A trader supplies a product to $n$ different companies (consumers) $\left\{l_{1}, \ldots, l_{j}, \ldots, l_{n}\right\}$ after delivery of that product from different $m$ manifacturers (producers) $\left\{k_{1}, \ldots, k_{i}, \ldots, k_{m}\right\}$ in quantities $c_{k_{i}, R}$ (for $1 \leq i \leq m$ ). Let the consumers (destinations) need this product in quantities of $c_{Q, l_{j}}$ (for $1 \leq j \leq n$ ).

Let $c_{k_{i}, l_{j}}$ be the intuitionistic fuzzy cost for transporting one unit quantity of the product from the $k_{i}$-th producer to the $l_{j}$-th consumer; $x_{k_{i}, l_{j}}$ - the number of units of the product, transported from $k_{i}$-th source to $l_{j}$-th destination and $c_{p l, l_{j}}$ (for $1 \leq j \leq n$ ) are limits to the transportation costs of the delivery a product from the $k_{i}$-th manifacturer to the $l_{j}$-th destination under form of IFPs.

All parameters, involved in the problem, are IFPs. For estimating the transportation costs in the form of IFPs, we can use the expert approach described in detail in [19]. Each expert needs to evaluate at least a part of the alternatives in terms of their performance with respect to each defined criterion. The experts is not sure about the transportation costs due the climatic and traffic conditions, or economic factors. He hesitates in prediction of the transportation cost due to changes in some uncontrollable factors. The transportation costs are evaluated as intuitionistic fuzzy numbers after a thorough discussion, interpreted by the intuitionistic fuzzy concept: these numbers express a "positive" and a "negative" evaluations, respectively. The reliability of the expert assessment (confidence in her/his evaluation with respect to each criterion) may be involved in the evaluation process. The purpose of the trader is how to satisfy the requests of the users so that the intuitionistic fuzzy transportation cost is minimum according to (2).

Let us formulate the mathematical model of the above problem:

$$
\begin{aligned}
& \text { An objective function: minimize } \sum_{i=1}^{m} \sum_{j=1}^{n} c_{k_{i}, l_{j}} x_{k_{i}, l_{j}} \\
& \text { Subject to: } \sum_{j=1}^{n} x_{k_{i}, l_{j}}=c_{k_{i}, R}, \quad i=1,2, \ldots, m \\
& \sum_{i=1}^{m} x_{k_{i}, l_{j}}=c_{Q, l_{j}}, \quad j=1,2, \ldots, n
\end{aligned}
$$

We add the constraint to the problem (3): $c_{p l, l_{j}}$, for $1 \leq j \leq$ $n$ - an intuitionistic fuzzy upper limit to the corresponding transportation cost of delivery a particular product from the $k_{i}$-th source to the $l_{j}$-th destination.

Note: The operations "addition" and "multiplication", used in the problem (3) are those for IFPs, defined in Sect. II.

The transportation costs of the problem (3) for delivery from a given manifacturer to a given user are entered in the cost IM $C$ :

$$
C[K, L]
$$

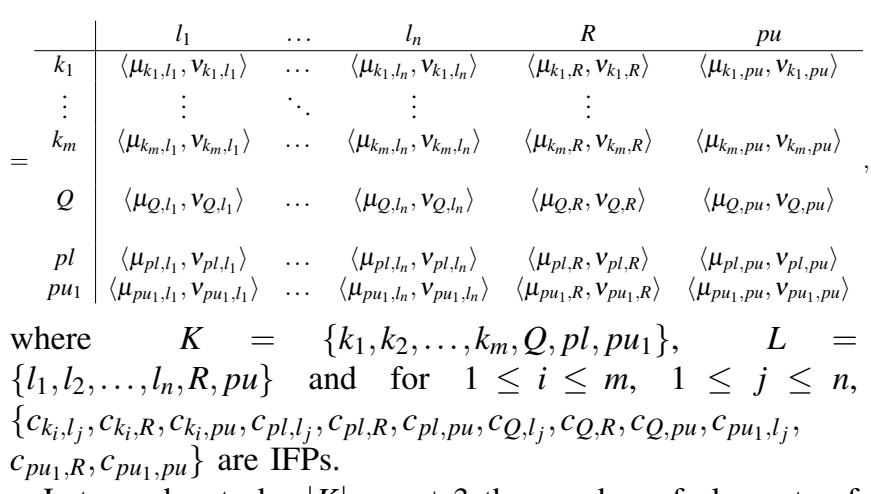

Let we denote by $|K|=m+3$ the number of elements of the set $K$; then $|L|=n+2$. We also define the IM

$$
X[K *, L *]=\begin{array}{c|ccccc} 
& l_{1} & \ldots & l_{j} & \ldots & l_{n} \\
\hline k_{1} & x_{k_{1}, l_{1}} & \cdots & x_{k_{1}, l_{j}} & \cdots & x_{k_{1}, l_{n}} \\
\vdots & \vdots & \ddots & \vdots & \ddots & \vdots \\
k_{m} & x_{k_{m}, l_{1}} & \ldots & x_{k_{m}, l_{j}} & \ldots & x_{k_{m}, l_{n}}
\end{array},
$$

$K *=\left\{k_{1}, k_{2}, \ldots, k_{m}\right\}, L *=\left\{l_{1}, l_{2}, \ldots, l_{n}\right\}$, and for $1 \leq i \leq m$, $1 \leq j \leq n: x_{k_{i}, l_{j}}=\left\langle\rho_{k_{i}, l_{j}}, \sigma_{k_{i}, l_{j}}\right\rangle$.

For the needs of the algorithm, let us we create the following auxiliary index matrices:

1) $S=\left[K, L,\left\{s_{k_{i}, l_{j}}\right\}\right]$, such that $S=C$ i.e. $\left(s_{k_{i}, l_{j}}=c_{k_{i}, l_{j}} \forall k_{i} \in\right.$ $\left.K, \forall l_{j} \in L\right)$;

2)

$$
D[K *, L *]=\begin{array}{c|ccccc} 
& l_{1} & \ldots & l_{j} & \ldots & l_{n} \\
\hline k_{1} & d_{k_{1}, l_{1}} & \cdots & d_{k_{1}, l_{j}} & \cdots & d_{k_{1}, l_{n}} \\
\vdots & \vdots & \ddots & \vdots & \ddots & \vdots \\
k_{m} & d_{k_{m}, l_{1}} & \ldots & d_{k_{m}, l_{j}} & \ldots & d_{k_{m}, l_{n}}
\end{array},
$$

where $K *=\left\{k_{1}, k_{2}, \ldots, k_{m}\right\}, L *=\left\{l_{1}, l_{2}, \ldots, l_{n}\right\}$, and for $i=$ $1, \ldots, m ; j=1, \ldots, n: \quad d_{k_{i}, l_{j}}=\{1$ or 2$\}$ depending on whether the elements $s_{k_{i}, l_{j}}$ of $S$ are crossed out with 1 or 2 lines. 3)

$$
R C\left[K *, e_{0}\right]=\begin{array}{c|c} 
& e_{0} \\
\hline k_{1} & r c_{k_{1}, e_{0}} \\
\vdots & \vdots \\
k_{m} & r c_{k_{m}, e_{0}}
\end{array},
$$

where $K *=\left\{k_{1}, k_{2}, \ldots, k_{m}\right\}$ and for $1 \leq i \leq m: \quad r c_{k_{i}, l_{j}}=$ $\{0$ or 1$\}$ depending on whether the $k_{i}$-th row of the matrix $S$ is crossed out or not.

4)

$$
C C\left[r_{0}, L *\right]=\begin{array}{c|ccccc} 
& l_{1} & \cdots & l_{j} & \cdots & l_{n} \\
\hline r_{0} & c c_{r_{0}, l_{1}} & \cdots & c c_{r_{0}, l_{j}} & \cdots & c c_{r_{0}, l_{n}}
\end{array}
$$

where $L *=\left\{l_{1}, l_{2}, \ldots, l_{n}\right\}$, and for $1 \leq j \leq n: c c_{k_{i}, l_{j}}=\{0$ or 1$\}$ depending on whether the $l_{j}$-th row of the matrix $S$ is crossed out or not.

5)

$$
R M\left[K /\left\{Q, p l, p u_{1}\right\}, R\right]=p r_{K /\left\{Q, p l, p u_{1}\right\}, R} C
$$

and

$$
C M\left[p u_{1}, L /\{R, p u\}\right]=p r_{p u_{1}, L /\{R, p u\}} C ;
$$


6) $U\left[K *, L *,\left\{u_{k_{i}, l_{j}}\right\}\right]$ and for $1 \leq i \leq m, 1 \leq j \leq n$ :

$$
u_{k_{i}, l_{j}}=\left\{\begin{array}{ll}
1, & \text { if } c_{k_{i}, l_{j}}<c_{p l, l_{j}} \\
\perp, & \text { otherwise }
\end{array} ;\right.
$$

When starting the algorithm, $r m_{k_{i}, R}=r c_{k_{i}, e_{0}}=c c_{r_{0}, l_{j}}=$ $c m_{p u_{1}, l_{j}}=0, u_{k_{i}, l_{j}}=\perp, x_{k_{i}, l_{j}}=\langle 0,1\rangle\left(\forall k_{i} \in K *, \forall l_{j} \in L *\right)$.

We will propose for the first time a new intuitionistic fuzzy approach for determining the optimal solution of the TP with intuitionistic fuzzy costs, demand and supply extending the zero point method ([2], [35], [34], [43]) and using the concepts of IMs and IFPs. In the program code was used a part of Microsoft Visual Studio.NET 2010 C project's.

Step 1. Let us create the IFIM $C$ for the given problem and then, convert it into a balanced one $\left(\sum_{i=1}^{m} c_{k_{i}, R}=\sum_{j=1}^{n} c_{Q, l_{j}}\right)$, if it is not.

The program executes the following operations:

- We define $2-D$ IMs as follows:

$$
\begin{gathered}
S_{1}[Q, L /\{R, p u\}]=p r_{Q, L /\{R, p u\}} C ; \\
S_{2}\left[K /\left\{Q, p l, p u_{1}\right\}, R\right]=p r_{K /\left\{Q, p u_{1}\right\}, R} C
\end{gathered}
$$

and let $\left\{k_{m+1}, l_{n+1}\right\} \notin K \cup L$.

By $L /\{R, p u\}$ let us denote the index set $L$ without the indices $R, p u$.

- If $\alpha_{K, \#_{q}}\left(S_{1}, l_{n+1}\right) \quad \supset_{v}\left[\frac{Q}{R} ; \perp\right]\left(\alpha_{L, \#_{q}}\left(S_{2}, l_{n+1}\right)\right)^{\prime} \quad$ (i.e. $\left.\sum_{i=1}^{m} c_{k_{i}, R}>\sum_{j=1}^{n} c_{Q, l_{j}}\right)$, then

introduce dummy column $l_{n+1}$ having all its costs as $\langle 0,1\rangle$ and execute operations for finding the demand at this dummy destination: $c_{Q, l_{n+1}}=\sum_{i=1}^{m} c_{k_{i}, R}-\sum_{j=1}^{n} c_{Q, l_{j}}$;

\{Let us define $2-D$ IMs $S_{3}, S_{4}, S_{5}$ such that

$$
\begin{gathered}
S_{3}=\alpha_{K, \#_{q}}\left(S_{1}, l_{n+1}\right)-{ }_{(\max , \min ))} \alpha_{L, \#_{q}}\left(\left[\frac{Q}{R} ; \perp\right]\left(S_{2}, l_{n+1}\right)\right)^{\prime} ; \\
S_{4}=\left[K /\left\{Q, p l, p u_{1}\right\},\left\{l_{n+1}\right\},\{\langle 0,1\rangle\}\right] ; \\
S_{5}=\left[K,\left\{l_{n+1}\right\},\left\{c_{k_{i}, l_{n+1}}\right\}\right]=S_{3} \oplus_{(\max , \min ))} S_{4} ;
\end{gathered}
$$

The new matrix of costs is obtained by carrying out the operation "matrix addition":

$C:=C \oplus_{(\max , \min ))} S_{5}$, go to Step 2. $\}$

- If $\left.\left.\left[\perp ; \frac{R}{Q}\right] \alpha_{K, \#_{q}}\left(S_{1}, k_{m+1}\right)\right)^{\prime} \subset_{v} \quad \alpha_{L, \#_{q}}\left(S_{2}, k_{m+1}\right)\right)^{\prime}$

$\sum_{i=1}^{m} c_{k_{i}, R}<\sum_{j=1}^{n} c_{Q, l_{j}}$ ), then

introduce dummy row $k_{m+1}$ having all its costs as $\langle 0,1\rangle$ and execute operations for finding the demand at this dummy destination: $c_{k_{m+1}, R}=\sum_{i=1}^{m} c_{k_{i}, R}-\sum_{j=1}^{n} c_{Q, l_{j}}$.

\{Let us define $2-D$ IMs $S_{3}, S_{4}, S_{5}$ such that

$$
\begin{gathered}
\left.S_{3}=\alpha_{K, \#_{q}}\left(C_{2}, k_{n+1}\right)-{ }_{(\max , \min ))}\left[\perp ; \frac{R}{Q}\right] \alpha_{L, \#_{q}}\left(C_{1}, k_{m+1}\right)\right)^{\prime} ; \\
S_{4}\left[\left\{k_{m+1}\right\}, L /\{Q, p u\},\{\langle 0,1\rangle\}\right] ;
\end{gathered}
$$

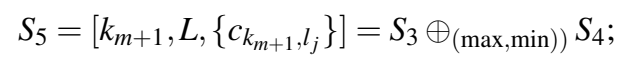

$C:=C \oplus_{(\max , \min ))} S_{5}$, go to Step 2. \}

Step 2. Checking the conditions for limiting the transportation costs

for (int $i=1 ; i<m ; i++$ )

for (int $j=1 ; j<n ; j++$ )

$$
\begin{gathered}
\left\{\text { If }\left(\left[\frac{k_{i}}{p l} ; \perp\right] p r_{p l, l_{j}} C\right) \supset_{v} p r_{k_{i}, l_{j}} C, \operatorname{then} u_{k_{i}, l_{j}}=1 .\right\} \\
E G=\operatorname{Index}_{(\perp)}(U) \\
=\left\{\left\langle k_{i_{1}}, l_{j_{1}}\right\rangle,\left\langle k_{i_{2}}, l_{j_{2}}\right\rangle, \ldots,\left\langle\left\langle k_{i_{\phi}}, l_{j_{\phi}}\right\rangle\right\} ;\right.
\end{gathered}
$$

for each $\left\langle k_{i}, l_{j}\right\rangle \in E G$, let us the element $s_{k_{i}, l_{j}}$ of $S$ is equal to $\langle 1,0\rangle[31] ;$

Go to Step 3.

Step 3. Determination of zero membership value - row level For each row of the matrix $S$, the smallest element is found in accordance with the relations (2) and is saved to the right of the row, in the column $p u$. The code uses the operation $A G I O$ for finding the indexes of the minimum elements of the row:

for (int $i=1 ; i<m ; i++$ )

for (int $j=1 ; j<n ; j++$ )

$\left\{\operatorname{AGIndex}_{\left\{(\min ) /\left(\min _{\square}\right) /\left(\min _{\odot}\right) /\left(\min _{R}\right)\right\}}\left(p r_{k_{i}, L /\{R, p u\}} S\right)=\left\langle k_{i}, l_{v_{j}}\right\rangle ;\right.$

If $p r_{k_{i}, l_{v_{j}}} S \subseteq_{v}\left(\left[\frac{k_{i}}{p l} ; \perp\right] p r_{p l, l_{v}} S\right)$, then

$$
\begin{gathered}
S_{6}\left[k_{i}, l_{v_{j}}\right]=p r_{k_{i}, l_{v}} S ; S_{7}=\left[\perp ; \frac{p u}{l_{v_{j}}}\right] S_{6} ; \\
\left.S:=S \oplus_{(\text {max }, \text { min })} S_{7} .\right\}
\end{gathered}
$$

Then from each element of the matrix $S$, subtract the smallest element in the same row:

for (int $i=0 ; i<m ; i++$ )

for (int $j=0 ; j<n ; j++$ )

$\left\{I O_{-(\max , \min )}\left(\left\langle k_{i}, l_{j}, S\right\rangle,\left\langle k_{i}, p u, p r_{K /\left\{Q, p l, p u_{1}\right\}} S\right\rangle\right)\right\}$;

Go to Step 4 .

Step 4. Determination of zero membership value - column level For each column of the matrix $S$, the smallest element is found in accordance with the relations (2). It is saved at the bottom of the column, in line $p u_{1}$ :

for (int $j=1 ; j<n ; j++$ )

$$
\begin{gathered}
\left\{\operatorname{AGIndex}_{\left\{(\min ) /\left(\min _{\square}\right) /\left(\min _{\odot}\right) /\left(\min _{R}\right)\right\}}\left(p r_{K /\left\{Q, p l, p u_{1}\right\}, l_{j}} S\right)\right. \\
=\left\langle k_{w_{i}}, l_{j}\right\rangle ;
\end{gathered}
$$

Let us create two 2-D IMs $S_{6}$ and $S_{7}$ :

$$
\begin{gathered}
S_{6}\left[k_{w_{i}}, l_{j}\right]=p r_{k_{w_{i}}, l_{j}} S ; S_{7}=\left[\frac{p u_{1}}{k_{w_{i}}} ; \perp\right] S_{6} ; \\
\left.S:=S \oplus_{(\max , \min )} S_{7} .\right\}
\end{gathered}
$$


for (int $j=1 ; j<n ; j++$ )

for (int $i=1 ; i<m ; i++$ )

$$
\left\{I O_{-(\max , \min )}\left(\left\langle k_{i}, l_{j}, S\right\rangle,\left\langle p u_{1}, l_{j}, p r_{p u_{1}, L /\{R, p u\}} S\right\rangle\right)\right\}
$$

Go to Step 5.

\section{Step 5. Optimality criterion}

1) Check if each quantity offered is less than or equal to the total quantity offered, whose reduced costs are with zero membership degrees.

for (int $i=1 ; i<m ; i++$ )

$$
\left\{\operatorname{Index}_{(\min \mu), k_{i}}(A)=\left\{\left\langle k_{i}, l_{v_{1}}\right\rangle, \ldots,\left\langle k_{i}, l_{v_{x}}\right\rangle, \ldots,\left\langle k_{i}, l_{v_{V}}\right\rangle ;\right.\right.
$$

We create $2-D$ IMs as follows:

$$
\begin{gathered}
G_{v_{1}}\left[k_{i}, l_{v_{1}}\right]=\operatorname{pr}_{k_{i}, l_{v_{1}}} C, \ldots, G_{v_{V}}\left[k_{i}, l_{v_{V}}\right]=p r_{k_{i}, l_{V}} C, \\
\text { and } G\left[k_{i}, R\right]=p r_{k_{i}, R} C ;
\end{gathered}
$$

If

$G\left[k_{i}, R\right] \subseteq_{v} G_{v_{1}}+{ }_{(\max , \min )} \ldots+_{(\max , \min )} G_{v_{x}}+\ldots{ }_{(\max , \min )} G_{v_{V}}$,

then go to Step 5.2.

else $\left\{R M\left[k_{i}, R\right]=1\right.$ and go to Step 6. $\}$

\}

2) Check if each required quantity is less than or equal to the total required quantity, whose reduced costs have zero membership degrees.

for (int $j=1 ; j<n ; j++$ )

$$
\left\{\operatorname{Index}_{(\min \mu), l_{j}}(A)=\left\{\left\langle k_{w_{1}}, l_{j}\right\rangle, \ldots,\left\langle k_{w_{y}}, l_{j}\right\rangle, \ldots,\left\langle k_{w_{W}}, l_{j}\right\rangle\right\} ;\right.
$$

We define $2-D$ IMs as follows:

$$
\begin{aligned}
G_{w_{1}}\left[k_{w_{1}}, l_{j}\right]= & p r_{k_{w_{1}}, l_{j}} C, \ldots, G_{w_{W}}\left[k_{w_{W}}, l_{j}\right]=p r_{k_{w_{W}}, l_{j}} C \\
& \text { and } G\left[p u_{1}, l_{j}\right]=p r_{p u_{1}, l_{j}} C
\end{aligned}
$$

If

$$
\begin{gathered}
G\left[p u_{1}, l_{j}\right] \\
\subseteq_{v} G_{w_{1}}+_{(\max , \min )} \ldots+_{(\max , \min )} G_{w_{y}}+\ldots+_{(\max , \min )} G_{w_{W}},
\end{gathered}
$$

then go to Step 8.

else $\left\{C M\left[p u_{1}, l_{j}\right]=1\right.$ go to Step 6.$\}$

\}

Step 6. Revise the cost IM All elements $\langle 0,1\rangle$ in the $S$ are crossed out with minimum number of lines (horizontal, vertical or both). If there is no element $\langle 0,1\rangle$ in a given row or column, then the element with the minimum degree of membership is crossed out from that row or column in the cost IM $S$ obtained in step 4. (omitting the unsatisfied supply and demand of 5.1 and 5.2 .

This step introduces IM $D[K *, L *]$, which has the same dimensions as the $X$ matrix. We use it to mark whether an element in the $S$ is crossed out with a horizontal or vertical line, or both.

If

$$
d_{k_{i}, l_{j}}=1,
$$

$s_{k_{i}, l_{j}}$ is crossed out with 1 line;

If

$$
d_{k_{i}, l_{j}}=2
$$

the $s_{k_{i}, l_{j}}$ element is covered with 2 lines.

We create two matrices $C C\left[r_{0}, L *\right]$ and $R C\left[K *, e_{0}\right]$, in which it is recorded that the element is covered by a line in a row or column in the $S$ matrix.

for (int $i=1 ; i<m ; i++$ )

for (int $j=1 ; j<n ; j++$ )

- If $s_{k_{i}, l_{j}}=\langle 0,1\rangle\left(\right.$ or $\left\langle k_{i}, l_{j}\right\rangle \in \operatorname{Index}_{(\min \mu), k_{i}}(S), r m_{k_{i}, R}=0$ and $d_{k_{i}, l_{j}}=0$,

then \{

$$
r c\left[k_{i}, e_{0}\right]=1 ; d_{k_{i}, l_{j}}=1 \forall l_{j} ; S_{\left(k_{i}, \perp\right)}
$$

\}

- If $\left\{s_{k_{i}, l_{j}}=\langle 0,1\rangle\left(\right.\right.$ or $\left\langle k_{i}, l_{j}\right\rangle \in \operatorname{Index}_{(\min \mu), k_{i}}(S), c m_{p u_{1}, l_{j}}=0$ and $\left.d_{k_{i}, l_{j}}=1\right\}$,

then \{

$$
d_{k_{i}, l_{j}}=2 ; c c_{r_{0}, l_{j}}=1 ; d_{k_{i}, l_{j}}=1 \forall k_{i} ; S_{\left(\perp, l_{j}\right)}
$$

\} .

Step 7. Develop the new revised cost IM We select the minimum IF cost of the $S$ using the relations (2), that is not crossed by the lines in Step 6, and subtract it from each of its uncovered elements, and we add it to each of its elements that is covered by two lines. We return to Step 5 .

$$
\operatorname{AGIndex}_{(\min , \max )}(S)=\left\langle k_{x}, l_{y}\right\rangle ;
$$

(that finds the smallest element index among the elements of the $S$ matrix.)

Subtract $S_{k_{x}, l_{y}}$ uncrossed each element of the matrix with reduced prices:

$$
I O_{-(\max , \min )}\left(\langle S\rangle,\left\langle k_{x}, l_{y}, S\right\rangle\right) .
$$

We add it to each element of $S$, which is crossed out by two lines, i.e. $d\left[k_{i}, l_{j}\right]=2$ :

for (int $i=1 ; i<m ; i++$ )

for (int $j=1 ; j<n ; j++$ )

if $d_{k_{i}, l_{j}}=2$ then create

$$
\begin{aligned}
S_{1}=p r_{k_{x}, l_{y}} C ; S_{2} & =p r_{k_{i}, l_{j}} C \oplus_{(\max , \min )}\left[\frac{k_{i}}{k_{x}} ; \frac{l_{j}}{l_{y}}\right] S_{1} ; \\
S & :=S \oplus_{(\max , \min )} S_{2} ;
\end{aligned}
$$

if $d_{k_{i}, l_{j}}=1$ then

$$
\left.S:=S \oplus_{(+)} p r_{k_{i}, l_{j}} C\right\}
$$

Go to Step 5 .

Step 8. Determination of a cell for allocation

1) Use relations (2) to select the largest IF cost in the IM 
$S$. If a tie exists, use any arbitrary tie-breaking choice. Let us denote this cell as $c_{k_{i *}, l_{j *}}$.

$$
\operatorname{AGIndex}_{(\max , \min )}(S)=\left\langle k_{x *}, l_{y *}\right\rangle \text {; }
$$

2) Select a single cost with zero degree of membership for allocation corresponding to $k_{i *}$-th row and/or $l_{j *}$-th column if exists and assigns the most possible to that cost cell and strike the satisfied IF supply or IF demand.

Let us $s_{k_{e}, l_{g}}=\min \left(s_{\operatorname{Index}(\min \mu), k_{* *}(A)}, s_{\operatorname{Index}(\min \mu), l_{j *}}\right)(A)$.

Then the minimum of the required and offered quantity is assigned to the corresponding $s_{k_{e}, l_{g}}$ cell and delete the row/column with exhausted required or offered quantity. So we find the reduced IM $S$.

We find minimum of $s_{k_{e}, R}$ and $s_{Q, l_{g}}$ by the operations:

We create the IMs $S_{8}\left[k_{e}, R\right]=p r_{k_{e}, R} S$ and $S_{9}\left[Q, l_{g}\right]=p r_{Q, l_{g}} S$; If $S_{8} \subseteq_{v}\left[\frac{k_{e}}{l_{g}} ; \frac{R}{Q}\right]\left(S_{9}\right)^{\prime}$ (i.e. $\left.\min \left(s_{k_{e}, R}, s_{Q, l_{g}}\right)=s_{k_{e}, R}\right)$, then

$$
\left\{X:=X \oplus_{(\max , \min )}\left[\perp ; \frac{l_{g}}{R}\right] S_{8} ;\right.
$$

We obtain a new matrix with dimensions

$(m+2) \times(n+2)$ by deleting the $k_{e}$-th row of the $S$ using the operation "reduction" $S_{\left(k_{e}, \perp\right)}$.

Let us create IM $S_{10}$ as follows:

$$
S_{10}\left[Q, l_{g}\right]=S_{9}-_{(\max , \min )}\left[\frac{Q}{R} ; \frac{l_{g}}{k_{e}}\right]\left(S_{8}\right)^{\prime} ;
$$

Then $\left.S:=S \oplus_{(\max , \min )} S_{10} ;\right\}$

If $S_{8} \supseteq_{v}\left[\frac{k_{i}}{l_{j}} ; \frac{R}{Q}\right]\left(S_{9}\right)^{\prime}$ (i.e. $\min \left(s_{k_{i}, R}, s_{Q, l_{j}}\right)=s_{Q, l_{j}}$ ), then $\{$ the IM $X$ changes with: $X:=X \oplus_{(\max , \min )}\left[\frac{k_{e}}{Q} ; \perp\right] S_{9}$.

We obtain a new matrix with dimensions $(m+3) \times(n+1)$ by reduction of the $l_{g}$-th column of $S$. Let us construct IM $S_{11}$ as follows:

$$
\begin{aligned}
S_{11}\left[k_{e}, R\right] & =S_{8}-_{(\max , \min )}\left[\frac{k_{e}}{l_{g}} ; \frac{R}{Q}\right]\left(S_{9}\right)^{\prime} ; \\
S & \left.:=S \oplus_{(\max , \min )} S_{11} ;\right\}
\end{aligned}
$$

Repeat Steps 8 until $|S|=6$ (all the required quantities are satisfied and all the offered quantities are exhausted), i.e. $S$ is reduced to the form

$S\left[K^{r}, L^{r}\right]=$\begin{tabular}{c|cc} 
reduced to the form & $R$ & $p u$ \\
\hline & $\left\langle\mu_{Q, R}, v_{Q, R}\right\rangle$ & $\left\langle\mu_{Q, p u}, v_{Q, p u}\right\rangle$ \\
$p l$ & $\left\langle\mu_{p l, R}, v_{p l, R}\right\rangle$ & $\left\langle\mu_{p l, p u}, v_{p l, p u}\right\rangle$ \\
$p u_{1}$ & $\left\langle\mu_{p u_{1}, R}, v_{p u_{1}, R}\right\rangle$ & $\left\langle\mu_{p u_{1}, p u}, v_{p u_{1}, p u}\right\rangle$
\end{tabular}$;$

Go to Step 9.

\section{Step 9.}

$$
\begin{gathered}
D=\operatorname{Index}_{\not} X \\
=\left\{\left\langle k_{i *_{1}}, l_{j *_{1}}\right\rangle, \ldots,\left\langle k_{i *_{f}}, l_{j *_{f}}\right\rangle, \ldots,\left\langle k_{i *_{\varphi}}, l_{j *_{\varphi}}\right\rangle\right\} .
\end{gathered}
$$

If the intuitionistic fuzzy feasible solution is degenerated (it contains less than $m+n-1$ (the total number of producers and consumers decreased by 1 ) occupied cells in the $X$ i.e. $|D|<m+n-1)$ [8] then increase the basic cells $x_{k_{i}, l_{j}}$ with one to which the minimum transportation cost corresponds.
Let us the recorded delivery of this cell is $\langle 0,1\rangle$. The IMs operations are:

If

$$
|D|<m+n-1
$$

then

$\left\{\right.$ AGIndex $\left\{(\min / \max ) /\left(\min _{\square} / \max _{\square}\right) /\left(\min _{\diamond} / \max _{\diamond}\right)\left(\min _{R} / \max _{R}\right)\right\}(\not \chi)(\notin D)(C)$

$$
=\left\langle k_{\alpha}, l_{\beta}\right\rangle
$$

$\left.x_{k_{a l}, l_{\beta}}=\langle 0,1\rangle\right\}$.

Go to Step 10.

\section{Step 10.}

for (int $i=1 ; i<m ; i++$ )

for (int $j=1 ; j<n ; j++$ )

If $x_{k_{i}, l_{j}} \neq\langle\perp, \perp\rangle$ and $\left\langle k_{i}, l_{j}\right\rangle \in E G$ then the problem has not solution [8] and the algorithm stop else

\{all the required and offered quantities are exhausted and the algorithm stop. The optimal basic solution $X_{o p t}\left[K *, L *,\left\{x_{k_{i}, l_{j}}\right\}\right]$ is obtained.

for (int $i=1 ; i<m ; i++$ )

for (int $j=1 ; j<n ; j++$ )

If $x_{k_{i}, l_{j}}=\langle\perp, \perp\rangle$ then $x_{k_{i}, l_{j}}=\langle 0,1\rangle$.

The optimal intuitionistic fuzzy transportation cost is:

$$
A G I O_{\oplus_{(\max , \min )}}^{1}\left(C_{\left(\left\{Q, p l, p u_{1}\right\},\{R, p u\}\right)} \otimes_{(\min , \max )} X_{o p t}\right)
$$

or

$$
A G I O_{\left.\oplus_{\left(\vee_{2}\right)}\right)}^{2}\left(C_{\left(\left\{Q, p l, p u_{1}\right\},\{R, p u\}\right)} \otimes_{\left(\wedge_{2}\right)} X_{o p t}\right),
$$

where $\vee_{2}$ and $\wedge_{2}$ are the operations from (1).

\section{AN EXAMPLE OF THE IFTP}

Let us extend the IFTP from [49]: A trader supplies a product to 4 different companies $\left\{l_{1}, l_{2}, l_{3}, l_{4}\right\}$. Let a product be produced at the manifacturers $\left\{k_{1}, k_{2}, k_{3}\right\}$ in quantities $c_{k_{i}, R}$ (for $1 \leq i \leq 3)$. Let the companies $\left(\left\{l_{1}, l_{2}, l_{3}, l_{4}\right\}\right)$ demand this product in an quantity of $c_{Q, l_{j}}$ (for $1 \leq j \leq 4$ ) and $c_{p l, l_{j}}$ (for $1 \leq j \leq 4)$ are intuitionistic fuzzy limits to the transportation costs of delivery a particular product from the $k_{i}$-th source to the $l_{j}$-th destination. The trader is not certain about the transportation costs, the demanded and supplied quantities due to several uncertainties. Let the cost $c_{k_{i}, l_{j}}$ for transporting one unit quantity of the product from the $k_{i}$-th producer to the $l_{j}$-th user is an IFP and is an element of IFIM $C[K, L]$

$$
\begin{aligned}
& C[K, L]=\left\{\begin{array}{c|cccc} 
& l_{1} & l_{2} & l_{3} & \ldots \\
\hline k_{1} & \langle 0.6,0.2\rangle & \langle 0.7,0.1\rangle & \langle 0.3,0.1\rangle & \ldots \\
k_{2} & \langle 0.5,0.3\rangle & \langle 0.4,0.1\rangle & \langle 0.5,0.1\rangle & \ldots \\
k_{3} & \langle 0.4,0.2\rangle & \langle 0.3,0.2\rangle & \langle 0.6,0.1\rangle & \ldots \\
Q & \langle 0.4,0.2\rangle & \langle 0.5,0.3\rangle & \langle 0.6,0.2\rangle & \ldots \\
p l & \langle 0.55,0.3\rangle & \langle 0.6,0.4\rangle & \langle 0.75,0.2\rangle & \ldots \\
p u_{1} & \langle\perp, \perp\rangle & \langle\perp, \perp\rangle & \langle\perp, \perp\rangle & \ldots
\end{array}\right. \\
& \begin{array}{cccc}
\ldots & l_{4} & R & p u \\
\ldots & \langle 0.8,0.1\rangle & \langle 0.5,0.2\rangle & \langle\perp, \perp\rangle
\end{array} \\
& \begin{array}{llll}
\ldots & \langle 0.3,0.2\rangle & \langle 0.7,0.1\rangle & \langle\perp, \perp\rangle
\end{array} \\
& \ldots \quad\langle 0.7,0.2\rangle \quad\langle 0.4,0.5\rangle \quad\langle\perp, \perp\rangle \\
& \text {.. }\langle 0.06,0.02\rangle \quad\langle\perp, \perp\rangle \quad\langle\perp, \perp\rangle \\
& \ldots \quad\langle\perp, \perp\rangle \quad\langle\perp, \perp\rangle \quad\langle\perp, \perp\rangle \\
& \ldots \quad\langle\perp, \perp\rangle \quad\langle\perp, \perp\rangle \quad\langle\perp, \perp\rangle
\end{aligned}
$$


Let $x_{k_{i}, l_{j}}$ is the number of units of the product, transported from the $k_{i}$-th producer to $l_{j}$-th destination (for $1 \leq i \leq 3$ and $1 \leq j \leq 4)$ and is an element of IFIM $X$ with initial elements $\langle\perp, \perp\rangle$. The trader wants to satisfy the required quantities of the users so that the intuitionistic fuzzy transportation cost is minimum.

\section{Solution of the problem:}

Step 1. The problem is balanced.

Step 2. Checking the conditions for limiting the transportation costs

for (int $i=1 ; i<m ; i++$ )

for (int $j=1 ; j<n ; j++$ )

\{If

$$
\left(\left[\frac{k_{i}}{p l} ; \perp\right] p r_{p l, l_{j}} C\right) \subset_{v} p r_{k_{i}, l_{j}} C
$$

then

$$
u_{k_{i}, l_{j}}=1
$$

\} .

The IM $C$ is transformed in:

$$
C[K, L]=\left\{\begin{array}{c|cccc} 
& l_{1} & l_{2} & l_{3} & \ldots \\
\hline k_{1} & \langle 0.6,0.2\rangle & \langle 1,0\rangle & \langle 0.3,0.1\rangle & \ldots \\
k_{2} & \langle 0.5,0.3\rangle & \langle 0.4,0.1\rangle & \langle 0.5,0.1\rangle & \ldots \\
k_{3} & \langle 0.4,0.2\rangle & \langle 0.3,0.2\rangle & \langle 0.6,0.1\rangle & \ldots \\
Q & \langle 0.4,0.2\rangle & \langle 0.5,0.3\rangle & \langle 0.6,0.2\rangle & \ldots \\
p l & \langle 0.55,0.3\rangle & \langle 0.6,0.4\rangle & \langle 0.75,0.2\rangle & \ldots \\
p u_{1} & \langle\perp, \perp\rangle & \langle\perp, \perp\rangle & \langle\perp, \perp\rangle & \ldots \\
& \ldots & & \\
& \ldots & & & \\
& \ldots & \langle 0.3,0.2\rangle & \langle 0.7,0.1\rangle & \langle\perp, \perp\rangle \\
& \ldots & \langle 0.7,0.2\rangle & \langle 0.4,0.5\rangle & \langle\perp, \perp\rangle \\
& \ldots & \langle 0.65,0.02\rangle & \langle\perp, \perp\rangle & \langle\perp, \perp\rangle \\
& \ldots & \langle\perp, \perp\rangle & \langle\perp, \perp\rangle & \langle\perp, \perp\rangle \\
\end{array}\right.
$$

Let us define IM $S=\left[K, L,\left\{s_{k_{i}, l_{j}}\right\}\right]$ such that $S=C$.

Step 3. Determination of zero membership value - row level In each row of the $S[K, L]$, the smallest element is found in accordance with the relation (2):

$$
\langle a, b\rangle \leq_{R}\langle c, d\rangle \text { iff } R_{\langle a, b\rangle} \geq R_{\langle c, d\rangle}
$$

and it is subtracted from all elements in the row and go to Step 4.

$$
S=\left\{\begin{array}{c|cccc} 
& l_{1} & l_{2} & l_{3} & \ldots \\
\hline k_{1} & \langle 0.3,0.3\rangle & \langle 0.7,0.1\rangle & \langle 0,0.2\rangle & \ldots \\
k_{2} & \langle 0.2,0.5\rangle & \langle 0.1,0.3\rangle & \langle 0.2,0.3\rangle & \ldots \\
k_{3} & \langle 0.1,0.4\rangle & \langle 0,0.4\rangle & \langle 0.3,0.3\rangle & \ldots \\
Q & \langle 0.4,0.2\rangle & \langle 0.5,0.3\rangle & \langle 0.6,0.2\rangle & \ldots \\
p l & \langle 0.55,0.3\rangle & \langle 0.6,0.4\rangle & \langle 0.75,0.2\rangle & \ldots \\
p u_{1} & \langle\perp, \perp\rangle & \langle\perp, \perp\rangle & \langle\perp, \perp\rangle & \ldots
\end{array}\right.
$$

$$
\begin{array}{cccc}
\ldots & l_{4} & R & p u \\
\hline \ldots & \langle 0.7,0.1\rangle & \langle 0.5,0.2\rangle & \langle 0.3,0.1\rangle \\
\ldots & \langle 0,0.4\rangle & \langle 0.7,0.1\rangle & \langle 0.3,0.2\rangle \\
\ldots & \langle 0.4,0.4\rangle & \langle 0.4,0.5\rangle & \langle 0.3,0.2\rangle \\
\ldots & \langle 0.06,0.02\rangle & \langle\perp, \perp\rangle & \langle\perp, \perp\rangle \\
\ldots & \langle 0.65,0.3\rangle & \langle\perp, \perp\rangle & \langle\perp, \perp\rangle \\
\ldots & \langle\perp, \perp\rangle & \langle\perp, \perp\rangle & \langle\perp, \perp\rangle
\end{array}
$$

Step 4. Determination of zero membership value - column level The smallest element is found for each column of the matrix $S[K, L]$ in accordance with the relation from (2)

$$
\langle a, b\rangle \leq_{R}\langle c, d\rangle \text { iff } R_{\langle a, b\rangle} \geq R_{\langle c, d\rangle}
$$

and it is subtracted from all elements in the corresponding column and go to Step 5.

$$
S=\left\{\begin{array}{c|cccc} 
& l_{1} & l_{2} & l_{3} & \ldots \\
\hline k_{1} & \langle 0.2,0.7\rangle & \langle 0.7,0.3\rangle & \langle 0,0.4\rangle & \ldots \\
k_{2} & \langle 0.1,0.9\rangle & \langle 0.1,0.7\rangle & \langle 0.2,0.5\rangle & \ldots \\
k_{3} & \langle 0,0.8\rangle & \langle 0,0.8\rangle & \langle 0.3,0.5\rangle & \ldots \\
Q & \langle 0.4,0.2\rangle & \langle 0.5,0.3\rangle & \langle 0.6,0.2\rangle & \ldots \\
p l & \langle 0.55,0.3\rangle & \langle 0.6,0.4\rangle & \langle 0.75,0.2\rangle & \ldots \\
p u_{1} & \langle 0.1,0.4\rangle & \langle 0,0.4\rangle & \langle 0,0.2\rangle & \ldots \\
\multicolumn{5}{c}{} \\
\ldots & \multicolumn{4}{c}{} \\
\ldots & l_{4} & R & p u & \\
\ldots & \langle 0.7,0.3\rangle & \langle 0.5,0.2\rangle & \langle 0.3,0.1\rangle & \\
\ldots & \langle 0,0.8\rangle & \langle 0.7,0.1\rangle & \langle 0.3,0.2\rangle & \\
\ldots & \langle 0.4,0.6\rangle & \langle 0.4,0.5\rangle & \langle 0.3,0.2\rangle & \\
\ldots & \langle 0.65,0.02\rangle & \langle\perp, \perp\rangle & \langle\perp, \perp\rangle & \\
\ldots & \langle 0,04\rangle & \langle\perp, \perp\rangle & \langle\perp, \perp\rangle & \\
& & \langle\perp, \perp\rangle & \langle\perp, \perp\rangle &
\end{array}\right.
$$

\section{Step 5. Optimality criterion}

1) Check if each required quantity is less than or equal to the total required quantity, whose reduced costs are with zero membership degrees.

2) Check id each quantity offered is less than or equal to the total quantity offered, whose reduced costs have zero membership degrees.

3) If 5.1 and 5.2 are satisfied then go to Step 8. else go to Step 6.

Step 6. Revise the cost IM Minimum number of lines (horizontal, vertical or both) are drawn to cover all elements $\langle 0,1\rangle$ in the $S$. If there is no element $\langle 0,1\rangle$ in a given row or column, then the element with the minimum degree of membership is crossed out from that row or column in the cost IM $S$ obtained in Step 4.

Step 7. Develop the new revised cost IM We select the minimum IF cost of the $S$ that is not crossed by the lines in Step 6., and subtract it from each of its uncovered elements, and we add it to each of its elements that is covered by two lines. We return to Step 5 .

The Steps 5., 6. and 7. are executed twice and then proceeds 
to Step 8. IM $S$ takes the following form after these steps:

$$
\begin{array}{r}
S=\left\{\begin{array}{c|cccc} 
& l_{1} & l_{2} & l_{3} & \ldots \\
\hline k_{1} & \langle 0,1\rangle & \langle 0.5,0.5\rangle & \langle 0,0.4\rangle & \ldots \\
k_{2} & \langle 0,1\rangle & \langle 0,1\rangle & \langle 0.28,0.45\rangle & \ldots \\
k_{3} & \langle 0,0.8\rangle & \langle 0,0.8\rangle & \langle 0.43,0.41\rangle & \ldots \\
Q & \langle 0.4,0.2\rangle & \langle 0.5,0.3\rangle & \langle 0.6,0.2\rangle & \ldots \\
p l & \langle 0.55,0.3\rangle & \langle 0.6,0.4\rangle & \langle 0.75,0.2\rangle & \ldots \\
p u_{1} & \langle 0.1,0.4\rangle & \langle 0,0.4\rangle & \langle 0,0.2\rangle & \ldots
\end{array}\right. \\
\left\{\begin{array}{ccccc}
\ldots & l_{4} & R & p u & \\
\ldots \ldots & \langle 0.6,0.4\rangle & \langle 0.5,0.2\rangle & \langle 0.3,0.1\rangle \\
\ldots & \langle 0,0.8\rangle & \langle 0.7,0.1\rangle & \langle 0.3,0.2\rangle \\
\ldots & \langle 0.46,0.54\rangle & \langle 0.4,0.5\rangle & \langle 0.3,0.2\rangle \\
\ldots & \langle 0.06,0.02\rangle & \langle\perp, \perp\rangle & \langle\perp, \perp\rangle \\
\ldots & \langle 0.65,0.3\rangle & \langle\perp, \perp\rangle & \langle\perp, \perp\rangle \\
\ldots & \langle 0,04\rangle & \langle\perp, \perp\rangle & \langle\perp, \perp\rangle
\end{array}\right.
\end{array}
$$

\section{Step 8.}

1) Use relations from (2) to select the largest IF cost in the IM $S$. Let us denote this cell as $c_{k_{i *}, l_{j *}}$.

2) Select a single cost with zero degree of membership for allocation corresponding to $k_{i *}$-th row and/or $l_{j *}$-th column if exists and determine the most possible to that cost cell and strike the satisfied IF supply or IF demand.

Steps 8 . is repeated three times until $|S|=6$ (all the demands are satisfied and all the supplies are exhausted).

Step 9. The intuitionistic fuzzy optimal solution, presented by the IM $X_{o p t}$ is non-degenerated, it includes 6 occupied cells. The IM $X_{o p t}$ has the following form:

$X_{\text {opt }}=\left\{\begin{array}{c|cccc} & l_{1} & l_{2} & l_{3} & l_{4} \\ \hline k_{1} & \langle 0,1\rangle & \langle 0,1\rangle & \langle 0.5,0.2\rangle & \langle 0,1\rangle \\ k_{2} & \langle 0.4,0.2\rangle & \langle 0.1,0.8\rangle & \langle 0.1,0.4\rangle & \langle 0.06,0.02\rangle \\ k_{3} & \langle 0,1\rangle & \langle 0.4,0.5\rangle & \langle 0,1\rangle & \langle 0,1\rangle\end{array}\right.$.

Step 10. The optimal intuitionistic fuzzy optimal solution $X_{o p t}\left[K *, L *,\left\{x_{k_{i}, l_{j}}\right\}\right]$ is obtained. The optimal intuitionistic fuzzy transportation cost is:

$A G I O_{\left.\oplus_{(\max , \min )}\right)}^{1}\left(C_{\left(\left\{Q, p l, p u_{1}\right\},\{R, p u\}\right)} \otimes_{(\min , \max )} X_{o p t}\right)=\langle 0.4,0.2\rangle$

or

$$
A G I O_{\left.\oplus_{\left(\vee_{2}\right)}\right)}^{2}\left(C_{\left(\left\{Q, p l, p u_{1}\right\},\{R, p u\}\right)} \otimes_{\left(\wedge_{2}\right)} X_{o p t}\right)=\langle 0.464,0.006\rangle .
$$

The degree of membership (acceptance) of this optimal solution is equal to 0.4 (or 0.464 ) and the its degree of nonmembership (non-acceptance) is equal to 0.2 (or 0.006).

Let us compare the results, obtained after application of IFZSMA [49] and IFZPM over IFTP, presented in the section IV. The optimal solution IM $X_{o p t}[K *, L *]$, obtained after application of IFZSM is as follows [49]:

$X_{\text {opt }}=\left\{\begin{array}{c|cccc} & l_{1} & l_{2} & l_{3} & l_{4} \\ \hline k_{1} & \langle 0,1\rangle & \langle 0,1\rangle & \langle 0.5,0.2\rangle & \langle 0,1\rangle \\ k_{2} & \langle 0.4,0.2\rangle & \langle 0.2,0.6\rangle & \langle 0.1,0.4\rangle & \langle 0.03,0.02\rangle \\ k_{3} & \langle 0,1\rangle & \langle 0.4,0.5\rangle & \langle 0,1\rangle & \langle 0,1\rangle\end{array}\right.$.

The optimal intuitionistic fuzzy cost of the IFTP is [49]:

$A G I O_{\oplus_{(\max , \min )}}^{1}\left(C_{\left(\left\{Q, p l, p u_{1}\right\},\{R, p u\}\right)} \otimes_{(\min , \max )} X_{o p t}\right)=\langle 0.4,0.2\rangle$

or

$$
A G I O_{\oplus_{\left(\vee_{2}\right)}}^{2}\left(C_{\left(\left\{Q, p l, p u_{1}\right\},\{R, p u\}\right)} \otimes_{\left(\wedge_{2}\right)} X_{o p t}\right)=\langle 0.475,0.005\rangle .
$$

The optimal solutions (4) and (7), obtained respectively by the IFZSM and the IFZPM, coincide.

The ranking function $R$, defined in (2), we can use to rank alternatives of decision-making process. For the obtained optimal solutions of IFZSM and IFZPM $R_{\langle 0.4 ; 0.2\rangle}=$ $0.42, R_{\langle 0.475 ; 0.005\rangle}=0.39$, and $R_{\langle 0.464 ; 0.006\rangle}=0.41$. When we use the pairs of operations $\langle\max , \min \rangle$ and $\langle\min , \max \rangle$ in (5), the optmal transportation cost after IFZSM and IFZPM coincide. When we use the pairs of operations $\vee_{2}$ and $\left\langle\wedge_{2}\right\rangle$ in (6), the optmal transportation cost after IFZPM is less than the optimal transportation cost after IFZPM.

The example illustrates the reliability of the proposed IFZPM.

\section{CONCLUSION}

In this paper it is proposed for the first time to extend the FZPM [2] to IFZPM for determining an optimal solution of a type of IFTP using the concepts of the IMs anf IFSs. The formulated IFTP has additional constraints: upper limits to the transportation costs. The proposed algorithm for solution of the IFTP is illustrated with a numerical example. The optimal solution of the problem in the example is compared with that obtained by the intuitionistic fuzzy zero suffix method (IFZSM). The advantages of the proposed algorithm is that it can be easy generalized to the multidimensional intuitionistic fuzzy TPs [22] and also can be applied to both the TP with crisp parameters and with intuitionistic fuzzy ones.

In the future, we will extend IFZPM to the multidimensional intuitionistic fuzzy TPs [22] and will apply the proposed approach for the TPs in different areas.

\section{REFERENCES}

[1] A. Edwuard Samuel, "Improved zero point method," Applied mathematical sciences, vol. 6 (109), 2012, pp. 5421-5426.

[2] A. Edwuard Samuel, M. Venkatachalapathy, "Improved zero point method for unbalanced FTPs," International Journal of Pure and Applied Mathematics, vol. 94 (3), 2014, pp. 419-424.

[3] A. Gani, A. Samuel, D. Anuradha, "Simplex type algorithm for solving fuzzy transportation problem," Tamsui Oxf. J. Inf. Math. Sci., vol. 27, 2011, pp. 89-98.

[4] A. Gani, S. Abbas, "A new average method for solving intuitionistic fuzzy transportation problem," International Journal of Pure and Applied Mathematics, vol. 93 (4), 2014, pp. 491-499.

[5] A. Kaur, A. Kumar, "A new approach for solving fuzzy transportation problems using generalized trapezoidal fuzzy numbers," Applied Soft Computing, vol. 12 (3), 2012, pp. 1201-1213.

[6] A. Kaur, J. Kacprzyk and A. Kumar, Fuzzy transportation and transshipment problems, Studies in fuziness and soft computing, vol. 385 , 2020.

[7] A. Patil, S. Chandgude, "Fuzzy Hungarian Approach for Transportation Model," International Journal of Mechanical and Industrial Engineering, vol. 2 (1), pp. 77-80, 2012.

[8] B. Atanassov, Quantitative methods in business management, Publ. houseTedIna, Varna; 1994. (in Bulgarian) 
[9] D. Dinagar, K. Palanivel, "On trapezoidal membership functions in solving transportation problem under fuzzy environment," Int. J. Comput Phys. Sci., vol. 1, 2009, pp. 1-12.

[10] E. Szmidt, J. Kacprzyk, "Amount of information and its reliability in the ranking of Atanassov's intuitionistic fuzzy alternatives," in: RakusAndersson, E., Yager, R., Ichalkaranje, N., Jain, L.C. (eds.), Recent Advances in Decision Making, SCI, Springer, Heidelberg, vol. 222, DOI: 10.1007/978-3-642-02187-9_2, 2009, pp. 7-19.

[11] F. Jimenez, J. Verdegay, "Solving fuzzy solid transportation problems by an evolutionary algorithm based parametric approach," European Journal of Operational Research, vol. 117 (3),1999, pp. 485-510.

[12] F. Hitchcock, "The distribution of a product from several sources to numerous localities," Journal of Mathematical Physics, vol. 20, 1941 , pp. 224-230.

[13] G. Dantzig, Application of the simplex method to a transportation problem, Chapter XXIII, Activity analysis of production and allocation, New York, Wiley, Cowles Commision Monograph, vol. 13, 359-373; 1951.

[14] G. Gupta, A. Kumar, M. Sharma, "A Note on A New Method for Solving Fuzzy Linear Programming Problems Based on the Fuzzy Linear Complementary Problem (FLCP)," International Journal of Fuzzy Systems, 2016, pp. 1-5.

[15] H. Arsham, A. Khan, "A simplex type algorithm for general transportation problems-An alternative to stepping stone," Journal of Operational Research Society, vol. 40 (6), 2017, pp. 581-590.

[16] H. Basirzadeh, "An approach for solving fuzzy transportation problem, "Appl. Math. Sci., vol. 5, 2011, pp. 1549-1566.

[17] K. Atanassov, "Intuitionistic Fuzzy Sets," VII ITKR Session, Sofia, 20-23 June 1983 (Deposed in Centr. Sci.-Techn. Library of the Bulg. Acad. of Sci., 1697/84) (in Bulgarian). Reprinted: Int. J. Bioautomation, vol. 20(S1), 2016, pp. S1-S6.

[18] K. Atanassov, "Generalized index matrices," Comptes rendus de l'Academie Bulgare des Sciences, vol. 40(11), 1987, pp. 15-18.

[19] K. Atanassov, On Intuitionistic Fuzzy Sets Theory, STUDFUZZ. Springer, Heidelberg, vol. 283; DOI:10.1007/978-3-642-29127-2, 2012.

[20] K. Atanassov, Index Matrices: Towards an Augmented Matrix Calculus. Studies in Computational Intelligence, Springer, Cham, vol. 573; DOI 10.1007/978-3-319-10945-9, 2014.

[21] K. Atanassov, "Intuitionistic Fuzzy Logics," Studies in Fuzziness and Soft Computing, Springer, vol. 351, DOI:10.1007/978-3-319-48953-7, 2017.

[22] K. Atanassov, "n-Dimensional extended index matrices Part 1," Advanced Studies in Contemporary Mathematics, vol. 28 (2), 2018, pp. 245-259.

[23] K. Atanassov, E. Szmidt, J. Kacprzyk, "On intuitionistic fuzzy pairs," Notes on Intuitionistic Fuzzy Sets, vol. 19 (3), 2013, pp. 1-13.

[24] K. Kathirvel, K. Balamurugan, "Method for solving fuzzy transportation problem using trapezoidal fuzzy numbers," International Journal of Engineering Research and Applications, vol. 2 (5), 2012, pp. 2154-2158

[25] K. Kathirvel, K. Balamurugan, "Method for solving unbalanced transportation problems using trapezoidal fuzzy numbers," International Journal of Engineering Research and Applications, vol. 3 ( 4), 2013 , pp. 2591-2596.

[26] L. Kantorovich, M. Gavyrin, Application of mathematical methods in the analysis of cargo flows, Coll. of articles Problems of increasing the efficiency of transport, M.: Publ. house AHSSSR, 110-138; 1949. (in Russian)

[27] L. Zadeh, Fuzzy Sets, Information and Control, vol. 8 (3), 338-353; 1965.

[28] M. Gen, K. Ida, Y. Li, E. Kubota, "Solving bicriteria solid transportation problem with fuzzy numbers by a genetic algorithm," Computers \& Industrial Engineering, vol. 29 (1), 1995, pp. 537-541.

[29] M. Purushothkumar, M. Ananthanarayanan, S. Dhanasekar, "Fuzzy zero suffix Algorithm to solve Fully Fuzzy Transportation Problems,' International Journal of Pure and Applied Mathematics, vol. 119 (9), 2018, pp. 79-88
[30] M. Shanmugasundari, K. Ganesan, "A novel approach for the fuzzy optimal solution of fuzzy transportation problem," International journal of Engineering research and applications, vol. 3 (1), 2013, pp. 14161424

[31] N. Lalova, L. Ilieva, S. Borisova, L. Lukov, V. Mirianov, A guide to mathematical programming, Science and Art Publishing House, Sofia; 1980 (in Bulgarian)

[32] P. Jayaraman, R. Jahirhussain, "Fuzzy optimal transportation problem by improved zero suffix method via Robust Ranking technique," International Journal of Fuzzy Mathematics and systems, vol. 3 (4), 2013, pp. 303-311.

[33] P. Kumar, R. Hussain, "A method for solving unbalanced intuitionistic fuzzy transportation problems," Notes on Intuitionistic Fuzzy Sets, vol. 21 (3), 2015, pp. 54-65.

[34] P. Ngastiti, B. Surarso, B. Sutimin, "Zero point and zero suffix methods with robust ranking for solving fully fuzzy transportation problems,' Journal of Physics: Conference Series, vol. 1022, 2018, pp. 1-10.

[35] P. Pandian, G. Natarajan, "A new algorithm for finding a fuzzy optimal solution for fuzzy transportation problems," Applied Mathematical Sciences, vol. 4, 2010, pp. 79- 90.

[36] R. Antony, S. Savarimuthu, T. Pathinathan, "Method for solving the transportation problem using triangular intuitionistic fuzzy number," International Journal of Computing Algorithm, vol. 03, 2014, pp. 590605 .

[37] R. Jahirhussain, P. Jayaraman, "Fuzzy optimal transportation problem by improved zero suffix method via robust rank techniques," International Journal of Fuzzy Mathematics and Systems (IJFMS), vol. 3, 2013, pp 303-311.

[38] R. Jahihussain , P. Jayaraman, "A new method for obtaining an optinal solution for fuzzy transportation problems," International Journal of Mathematical Archive, vol. 4 (11), 2013, pp. 256-263.

[39] S. Chanas, W. Kolodziejckzy, A. Machaj, "A fuzzy approach to the transportation problem," Fuzzy Sets and Systems, vol. 13, 1984, pp 211-221.

[40] S. Dhanasekar, S. Hariharan, P. Sekar, "Fuzzy Hungarian MODI Algorithm to solve fully fuzzy transportation problems," Int. J. Fuzzy Syst., vol. 19 (5), 2017, pp. 1479-1491.

[41] S. Liu, C. Kao, "Solving fuzzy transportation problems based on extension principle," Eur. J. Oper. Res., vol. 153, 2004, pp. 661-674.

[42] S. Mohideen, P. Kumar, "A Comparative Study on Transportation Problem in Fuzzy Environment," International Journal of Mathematics Research, vol. 2 (1), 2010, pp. 151-158.

[43] T. Karthy, K. Ganesan, "Revised improved zero point method for the trapezoidal fuzzy transportation problems," AIP Conference Proceedings, 2112, 020063, 2019, pp. 1-8.

[44] V. Sudhagar, V. Navaneethakumar, "Solving the Multiobjective two stage fuzzy transportation problem by zero suffix method," Journal of Mathematics Research,vol. 2 (4), 2010, pp. 135-140.

[45] V. Traneva, "Internal operations over 3-dimensional extended index matrices," Proceedings of the Jangjeon Mathematical Society, vol. 18 (4), 2015, pp. 547-569.

[46] V. Traneva, S. Tranev, V. Atanassova, "An Intuitionistic Fuzzy Approach to the Hungarian Algorithm," in: G. Nikolov et al. (Eds.): NMA 2018, LNCS 11189, Springer Nature Switzerland, AG, 2019, pp. 1-9, DOI: 10.1007/978-3-030-10692-8_19.

47] V. Traneva, S. Tranev, M. Stoenchev, K. Atanassov, "Scaled aggregation operations over two- and three-dimensional index matrices," Soft computing, vol. 22, 2019, pp. 5115-5120, DOI: 10.1007/s00500-018-3315-6.

48] V. Traneva, S. Tranev, Index Matrices as a Tool for Managerial Decision Making, Publ. House of the Union of Scientists, Bulgaria; 2017 (in Bulgarian)

[49] V. Traneva, S. Tranev, "An Intuitionistic fuzzy zero suffix method for solving the transportation problem," in: Dimov I., Fidanova S. (eds) Advances in High Performance Computing. HPC 2019, Studies in computational intelligence, Springer, Cham, vol. 902, DOI: 10.1007/978-3030-55347-0 7, 2020 . 